\title{
Hamiltonian Structure, Soliton Solution and Conservation Laws for a New Fifth-Order Nonlinear Evolution Equation Which Describes Pseudo-Spherical Surfaces
}

\author{
S. M. Sayed ${ }^{1 *}$, N. O. Al-Atawi ${ }^{2}$ \\ ${ }^{1}$ Mathematics Department, Faculty of Science, Beni-Suef University, Beni-Suef, Egypt \\ ${ }^{2}$ Mathematics Department, Faculty of Science, University of Tabouk (UT), KSA \\ Email: ^smsayed71@gmail.com, *S_M_Sayed71@yahoo.com
}

How to cite this paper: Sayed, S.M. and Al-Atawi, N.O. (2017) Hamiltonian Structure, Soliton Solution and Conservation Laws for a New Fifth-Order Nonlinear Evolution Equation Which Describes Pseudo-Spherical Surfaces. American Journal of Computational Mathematics, 7, 166-174.

https://doi.org/10.4236/ajcm.2017.72015

Received: May 10, 2017

Accepted: June 19, 2017

Published: June 22, 2017

Copyright $\odot 2017$ by authors and Scientific Research Publishing Inc. This work is licensed under the Creative Commons Attribution International License (CC BY 4.0).

http://creativecommons.org/licenses/by/4.0/

\begin{abstract}
In this paper, we shall show that the Hamiltonian structure can be defined for any nonlinear evolution equations which describe surfaces of a constant negative curvature, so that the densities of conservation laws can be considered as corresponding Hamiltonians. This paper obtains the soliton solution and conserved quantities of a new fifth-order nonlinear evolution equation by the aid of inverse scattering method.
\end{abstract}

\section{Keywords}

Nonlinear Evolution Equations, Conservation Laws, Pseudo-Spherical Surfaces

\section{Introduction}

The notion of pseudo-spherical surfaces (pss) (surfaces of a constant negative curvature $k \equiv-1$ ) appeared in geometry in the middle of the nineteenth century. It was an important step in the development of mathematics. Pss become the final factor in the visual interpretation of non-Euclidean hyperbolic geometry discovered by Klingenberg [1]. The further development of mathematics found a close connection between pss and theory of nets, theory of solitons, attractors, some nonlinear evolution equations (NLEEs) of mathematical physics, Bäcklund transformations (BTs), and so on [2] [3] [4] [5]. The connection between geometry and the nonlinear partial differential equations (NLPDEs) has been studied in mathematical physics for more than a century. For instance, the classical Liouville equation describes minimal surfaces in the 
space $E^{3}$, and the sine Gordon equation is related to the geometry of pss, i.e., surfaces with a negative Gaussian curvature [6] [7] [8] [9].

Conservation law plays a vital role in the study of nonlinear evolution equations, particularly with regard to integrability, linearization and constants of motion. In the present paper, it is shown that infinitely many conservation laws for certain nonlinear evolution equations are systematically constructed with symbolic computation in a simple way from the Riccati form of the Lax pair. Noting that the Lax pairs investigated here are associated with different linear systems, including the generalized Kaup-Newell (KN) spectral problem, the generalized Ablowitz-Kaup-Newell-Segur (AKNS) spectral problem, the generalized AKNS-KN spectral problem and a recently proposed integrable system. Therefore, the power and efficiency of this systematic method are well understood, and we expect it may be useful for other nonlinear evolution models, even higher-order and variable-coefficient ones [10]-[15].

Hamiltonians are of great importance in their own right and have found a remarkable number of applications in both physics and mathematics. Hamiltonians play a central role in the field of integrable systems and also play a fundamental role in several others areas of mathematics and physics. Hamiltonians are often referred to as the master integrable system. Hamiltonians provide as with a means of generating and classifying many integrable systems and they also give a unified geometrical framework in which to analyze them. Moreover, in the context of the inverse scattering transform, an integrable equation admits well-behaved solutions obtained via the related linear problems [16]-[22].

The main aim of this paper is to use the BTs in the construction of exact soliton solutions for a new fifth-order nonlinear evolution equation which describes pss. An infinite number of conservation laws are derived for a new fifth-order nonlinear evolution equation just mentioned using the corresponding Hamiltonians.

The latter yields directly the curvature condition (Gaussian curvature equal to -1 , corresponding to pseudo-spherical surfaces). This geometrical method allows some further generalizations of the work on conservation laws given by Khater et al. [23]. An infinite number of conservation laws for a new fifth-order nonlinear evolution equation are derived in this way.

The paper is organized as follows. In Section 2, we introduce the inverse scattering method and apply the geometrical method to obtain Hamiltonian structure for any nonlinear evolution equations which describe surfaces of a constant negative curvature. In Section 3, a new exact soliton solution and the corresponding Hamiltonians are obtained for a new fifth-order nonlinear evolution equation. Section 4 contains the conclusion.

\section{Hamiltonian Structure}

The inverse scattering transform method allows one to linearize a large class of nonlinear evolution equations and can be considered as a nonlinear version of the Fourier transform [24] [25] [26] [27] [28]. An essential prerequisite of 
inverse scattering transform method is the association of the nonlinear evolution equation with a pair of linear problems (Lax pair), a linear eigenvalue problem, and a second associated linear problem, such that the given equation results as a compatibility condition between them [29] [30] [31] [32] [33]. Consider the following AKNS eigenvalues problem:

$$
\psi_{x}=P \psi, \quad \psi_{t}=Q \psi,
$$

where $\psi=\left(\begin{array}{l}\psi_{1} \\ \psi_{2}\end{array}\right), \quad P$ and $Q$ are two $2 \times 2$ null-trace matrices

$$
P=\left(\begin{array}{cc}
\frac{\eta}{2} & q \\
r & -\frac{\eta}{2}
\end{array}\right), \quad Q=\left(\begin{array}{cc}
A & B \\
C & -A
\end{array}\right),
$$

and $\eta$ is a parameter independent of $x$ and $t$, while $q$ and $r$ are assumed to be functions of $x$ and $t$. From Equations (1) and (2), we get the following scattering problem:

$$
\begin{aligned}
& \psi_{1 x}=\frac{\eta}{2} \psi_{1}+q \psi_{2}, \\
& \psi_{2 x}=r \psi_{1}-\frac{\eta}{2} \psi_{2},
\end{aligned}
$$

in which eignfunctions $\psi_{1}$ and $\psi_{2}$ evolve in time according to

$$
\begin{aligned}
& \psi_{1 t}=A(x, t ; \eta) \psi_{1}+B(x, t ; \eta) \psi_{2}, \\
& \psi_{2 t}=C(x, t ; \eta) \psi_{1}-A(x, t ; \eta) \psi_{2} .
\end{aligned}
$$

The integrability conditions reads

$$
P_{t}-Q_{x}+[P, Q]=0
$$

or in component form

$$
\begin{aligned}
& A_{x}=q C-r B, \\
& q_{t}-2 A q-B_{x}+\eta B=0, \\
& C_{x}=r_{t}+2 A r-\eta C,
\end{aligned}
$$

Konno and Wadati [34] introduced the function

$$
\Gamma=\frac{\psi_{1}}{\psi_{2}} .
$$

Differentiating Equation (7) with respect to $x$ and $t$, respectively, and using Equations (3), (4) and (7), then Equation (1) are reduced to the Riccati equations:

$$
\frac{\partial \Gamma}{\partial x}=\eta \Gamma-r \Gamma^{2}+q, \frac{\partial \Gamma}{\partial t}=2 A \Gamma-C \Gamma^{2}+B .
$$

Now we construct a transformation $\Gamma^{\prime}$ satisfies the potential and then deduce a BTs for the considered nonlinear evolution equation

$$
u^{\prime}=u_{0}+f(\Gamma, \eta),
$$

where $u_{0}$ is the old solution and $u^{\prime}$ is a new solution corresponding non- 
linear evolution equation. In order to determine conserved densities and fluxes, we can written Equation (8) in the form

$$
C \frac{\partial \Gamma}{\partial x}-r \frac{\partial \Gamma}{\partial t}=(q C-B r)+(\eta C-2 A r) \Gamma .
$$

Adding $-r_{t} \Gamma$ to both sides and using Equation (6), then Equation (10) takes the form of conservation laws

$$
\frac{\partial(r \Gamma)}{\partial t}=\frac{\partial(-A+C \Gamma)}{\partial x},
$$

where $r \Gamma$ are conserved densities and $(-A+C \Gamma)$ are fluxes.

From Equation (3), I obtain

$$
\frac{\psi_{2 x}}{\psi_{2}}=-\frac{\eta}{2}+r \Gamma
$$

then

$$
\ln \psi_{2}=-\left(\frac{\eta}{2}\right) x+\int r \Gamma \mathrm{d} x
$$

By rearranged the first equation of Equation (8) to take the form

$$
\eta(r \Gamma)=-r q+(r \Gamma)^{2}-r\left(\frac{r \Gamma}{r}\right)_{x},
$$

then, I can expand $r \Gamma$ into a power series in inverse power of $\eta$ as follows [28]

$$
r \Gamma(x, t ; \eta)=\sum_{n=1}^{\infty} \phi_{n}(x, t) \eta^{-n}
$$

By the same way, I expand $\ln \psi_{2}$ into a power series in inverse power of $\eta$ so that

$$
\ln \psi_{2}=H_{0}+\sum_{l=1}^{\infty} H_{l} \eta^{-l},
$$

where $H_{0}$ and $H_{l}$ are Hamiltonians (conserved quantities), by substituting (15) into (14), the following system of conservation laws appears

$$
\sum_{n=1}^{\infty} \phi_{n}(x, t) \eta^{-n+1}=-r q+\left(\sum_{n=1}^{\infty} \phi_{n}(x, t) \eta^{-n}\right)^{2}+r\left(\sum_{n=1}^{\infty}\left(\frac{\phi_{n}(x, t)}{r}\right)_{x} \eta^{-n}\right) .
$$

Now equate powers of $\eta$ on both sides of this expression to produce the set of recursions,

$$
\phi_{1}(x, t)=-q r, \quad \phi_{2}(x, t)=-r q_{x}, \phi_{n+1}=\sum_{k=1}^{n-1} \phi_{k}(x, t) \phi_{n-k}(x, t)+r\left(\frac{\phi_{n}(x, t)}{r}\right)_{x}, n \geq 2 .
$$

Then, by equate powers of $\eta$ on both sides of Equations (13), (15) and (16), we obtain the infinite number of Hamiltonians may explicitly be determined in terms of smooth real functions $\phi_{n}(x, t)$ and their derivatives, as follows

$$
H_{0}=-\left(\frac{\eta}{2}\right) x, \quad H_{1}=\int \phi_{1} \mathrm{~d} x=-\int q r \mathrm{~d} x,
$$




$$
H_{2}=\int \phi_{2} \mathrm{~d} x=-\int r q_{x} \mathrm{~d} x,
$$

Hamiltonians in general form

$$
H_{n+1}(x, t)=\int\left(\sum_{k=1}^{n-1} \phi_{k}(x, t) \phi_{n-k}(x, t)+r\left(\frac{\phi_{n}(x, t)}{r}\right)_{x}\right) \mathrm{d} x, \quad n \geq 2 .
$$

The explicit expressions of the first few order Hamiltonians are

$$
\begin{gathered}
H_{3}=\int \phi_{3} \mathrm{~d} x=\int\left(q^{2} r^{2}-r q_{x x}\right) \mathrm{d} x, \quad H_{4}=\int \phi_{4} \mathrm{~d} x=\int\left(4 r^{2} q q_{x}+r q^{2} r_{x}-r q_{3 x}\right) \mathrm{d} x, \\
H_{5}=\int \phi_{5} \mathrm{~d} x=\int\left[6 r^{2} q q_{2 x}+6 r q r_{x} q_{x}+4 r^{2} q_{x}^{2}+r q^{2} r_{2 x}-2 q^{3} r^{3}-r q_{4 x}\right] \mathrm{d} x .
\end{gathered}
$$

The procedure is clarified in the following example.

\section{Soliton Solution and an Infinite Number of Conserved Quantities for a New Fifth-Order Nonlinear Evolution Equation}

Now we consider a new fifth-order nonlinear evolution equation

$$
u_{t}=u_{5 x}-\frac{5}{2} u^{2} u_{3 x}-10 u u_{x} u_{2 x}-\frac{5}{2} u_{x}^{3}+\frac{15}{8} u^{4} u_{x}
$$

for $u(x, t)$ which describes a pss. There exist functions $f_{i j}, 1 \leq i \leq 3$, $1 \leq j \leq 2$, which depend on $u(x, t)$ and its derivatives such that, for any solution $u$ of the evolution equation, $f_{i j}$ satisfy (5). For Equation (20) we consider the functions defined by [33]

$$
\begin{gathered}
f_{11}=u, \quad f_{12}=u_{4 x}+\left(\frac{-5}{2} u^{2}-3 \eta^{2}\right) u_{2 x}-\frac{5}{2} u u_{x}^{2}+\frac{3}{8} u^{5}+\frac{3}{2} \eta^{2} u^{3}+9 \eta^{4} u, \\
f_{21}=\eta, \quad f_{22}=-\eta\left(2 u_{3 x}-3 u^{2} u_{x}-6 \eta^{2} u_{x}+u u_{2 x}-\frac{1}{2} u_{x}^{2}-\frac{3}{8} u^{4}-\frac{3}{2} \eta^{2} u^{2}-9 \eta^{4}\right), \\
f_{31}=2 \eta, \quad f_{32}=-\eta\left(u_{3 x}-32 u^{2} u_{x}-3 \eta^{2} u_{x}+2 u u_{2 x}-u_{x}^{2}-\frac{3}{4} u^{4}-3 \eta^{2} u^{2}-18 \eta^{4}\right) .
\end{gathered}
$$

For any solution $u(x, t)$ of a new fifth-order evolution Equation (20), the matrices $P$ and $Q$ are

$$
\begin{gathered}
P=\left(\begin{array}{cc}
\frac{\eta}{2} & \frac{u}{2}-\eta \\
\frac{u}{2}+\eta & -\frac{\eta}{2}
\end{array}\right), \\
Q=\left(\begin{array}{cc}
\frac{f_{22}}{2} & \frac{f_{12}-f_{32}}{2} \\
\frac{f_{12}+f_{32}}{2} & -\frac{f_{22}}{2}
\end{array}\right),
\end{gathered}
$$

where $f_{22}, f_{12}$ and $f_{32}$ defined by (21). The above matrices $P, Q$ satisfy the Equation (5). Then the first equation of (8) becomes

$$
\frac{\partial \Gamma}{\partial x}=\frac{\eta}{2}\left(\Gamma-\Gamma^{2}-1\right)+\frac{u}{2}\left(1-\Gamma^{2}\right)
$$

If we choose $\Gamma^{\prime}$ and $u^{\prime}$ as 


$$
\begin{gathered}
\Gamma^{\prime}=\frac{1}{\Gamma}, \\
u^{\prime}=-u+4 \frac{\partial}{\partial x} \tanh ^{-1} \Gamma,
\end{gathered}
$$

then $\Gamma^{\prime}$ and $u^{\prime}$ satisfies Equation (24).

Now we shall choose some known solutions of the above a new fifth-order evolution equation and substitute these solutions into the corresponding matrices $P$ and $Q$. Next, we solve Equations (3) for $\psi_{1}$ and $\psi_{2}$. Then, by (9) and the corresponding BT we shall obtain the new solutions for a new fifth order evolution equation. I choose the known solution is a constant $u_{0}$, then substitute $u=u_{0}$ into the matrices $P$ and $Q$ in (22) and (23), then by (1) we have

$$
\mathrm{d} \psi=\psi_{X} \mathrm{~d} x+\psi_{t} \mathrm{~d} t=P \psi \mathrm{d} \rho,
$$

where

$$
\begin{gathered}
P=\left(\begin{array}{cc}
\frac{\eta}{2} & \frac{u_{0}}{2}-\eta \\
\frac{u_{0}}{2}+\eta & -\frac{\eta}{2}
\end{array}\right), \\
\rho=x+\alpha t, \quad \alpha=\frac{3 u_{0}^{4}}{8}+\frac{3 \eta^{2} u_{0}^{2}}{2}+9 \eta^{4} .
\end{gathered}
$$

The solution of Equation (27) is

$$
\psi=(\exp \rho P) \psi_{0}=\left(I+\rho P+\frac{\rho^{2} P^{2}}{2 !}+\frac{\rho^{3} P^{3}}{3 !}+\cdots\right) \psi_{0},
$$

where $\psi_{0}$ is a constant column vector. The solution (30) takes the following form:

$$
\psi=\left[\begin{array}{cc}
\cosh \alpha \rho+\frac{\eta}{2 \alpha} \sinh \alpha \rho & \left(1-\frac{\eta}{\alpha}\right) \sinh \alpha \rho \\
\left(1+\frac{\eta}{\alpha}\right) \sinh \alpha \rho & \cosh \alpha \rho-\frac{\eta}{2 \alpha} \sinh \alpha \rho
\end{array}\right] \psi_{0}
$$

Now, we choose $\psi_{0}=\left(\begin{array}{l}1 \\ 0\end{array}\right)$, in (31) and use (7) and the BT (26); we obtain the new solution class of the new fifth-order evolution Equation (20) corresponding to the known constant solution $u_{0}$ as follows

$$
u=-u_{0}-\frac{4 a \alpha \operatorname{csch}^{2} \alpha \rho}{1-(b+a \operatorname{coth} \alpha \rho)^{2}}
$$

where $a=\frac{\alpha}{\alpha+\eta}, b=\frac{\eta}{2 \alpha+2 \eta}$. If we choose $\psi_{0}=\left(\begin{array}{l}0 \\ 1\end{array}\right)$ in (31), we will obtain another solution. Obviously all of these solutions are traveling waves with velocity $\alpha=\frac{3 u_{0}^{4}}{8}+\frac{3 \eta^{2} u_{0}^{2}}{2}+9 \eta^{4}$.

From Equations (19) and (22) the first few order Hamiltonians are deter- 
mined by the relation

$$
H_{0}=-\left(\frac{\eta}{2}\right) x, H_{1}=-\int\left(\frac{u^{2}}{4}-\eta^{2}\right) \mathrm{d} x, H_{2}=-\int \frac{u_{x}}{2}\left(\frac{u}{2}+\eta\right) \mathrm{d} x, \text { etc. }
$$

From the solution $u(x, t)$ of a new fifth-order evolution equation. This Hamiltonians (conserved quantities) given by the relation

$$
\begin{aligned}
H_{0}= & -\left(\frac{\eta}{2}\right) x, \\
H_{1}=\left(\eta^{2}-\frac{u_{0}^{2}}{4}\right) x+\frac{4 \alpha^{2} \eta \sinh 2 \alpha \rho}{(2 \alpha+\eta)(-4 \alpha-\eta+\eta \operatorname{coth} 2 \alpha \rho)} & \\
& -\frac{2 \sqrt{2} \sqrt{\alpha}\left(4 \alpha^{2}-2 u_{0} \alpha+\alpha \eta-u_{0} \eta\right) \operatorname{arctanh}\left(\sqrt{1+\frac{\eta}{2 \alpha}} \tanh \alpha \rho\right)}{(2 \alpha+\eta)^{\frac{3}{2}}}, \\
H_{2}= & \frac{-4 \alpha^{2}\left(8 \alpha^{2}-4 u_{0} \alpha+8 \alpha \eta-u_{0} \eta+2 \eta^{2}+\left(u_{0}-2 \eta\right) \eta \cosh 2 \alpha \rho\right)}{(4 \alpha+\eta-\eta \cosh 2 \alpha \rho)^{2}}
\end{aligned}
$$

etc.

\section{Conclusion}

We may hope to find the relationship between the conserved quantities and pss. The conserved quantities play a central role in the field of integrable systems and also play a fundamental role in several other areas of mathematics and physics [35]. In addition, the conserved quantities are a rich source of integrable systems suggested by the fact that they are the compatibility condition of an associated linear problem which admits enormous freedom if one allows the associated gauge algebra to be arbitrary [36].

\section{Acknowledgements}

The author would like to thank the anonymous referees for these helpful comments. The author thinks that revising the paper according to its suggestions will be quite straightforward.

\section{References}

[1] Klingenberg, W. (1982) Riemannian Geometry. Walter de Gruyter, Berlin, New York.

[2] Chuu-Lian, T. and Uhlenbeck, K. (1999) Introduction to Surveys in Differential Geometry: Integrable Systems. Journal of Differential Geometry, 4, 5-21.

[3] Abolwitz, M.J. and Clarkson, P.A. (1991) Solitons, Nonlinear Evolution Equations and Inverse Scattering. Cambridge University Press, Cambridge. https://doi.org/10.1017/CBO9780511623998

[4] Tenenblat, K. and Terng, C.L. (1980) Bäcklund's Theorem for n-Dimensional Submanifolds of $R^{2 n-1}$. Annals of Mathematics, 111, 477-481. https://doi.org/10.2307/1971105

[5] Chern, S.S. and Terng, C.L. (1980) An Analogue of Bäcklund Theorem in Affine Geometry. Rocky Mountain Mathematics Consortium, 10, 12105-1211. 
[6] Chern, S.S. and Tenenblat, K. (1986) Pseudospherical Surfaces and Evolution Equations. Studies in Applied Mathematics, 74, 1-7. https://doi.org/10.1002/sapm198674155

[7] Cavalcante, J.A. and Tenenblat, K. (1988) Conservation Laws for Nonlinear Evolution Equations. Journal of Mathematical Physics, 29, 1044-1053.

https://doi.org/10.1063/1.528020

[8] Beals, R., Rabelo, M. and Tenenblat, K. (1989) Bäcklund Transformations and Inverse Scattering Solutions for Some Pseudo-Spherical Surfaces. Studies in Applied Mathematics, 81, 125-133. https://doi.org/10.1002/sapm1989812125

[9] Reyes, E.G. (2000) Conservation Laws and Calapso-Guichard Deformations of Equations Describing Pseudo-Spherical Surfaces. Journal of Mathematical Physics, 41, 2968-2979. https://doi.org/10.1063/1.533284

[10] Reyes, E.G. (2001) On Geometrically Integrable Equations and Hierarchies of Pseudo-Spherical Type. Contemporary Mathematics, 285, 145-151. https://doi.org/10.1090/conm/285/04740

[11] Yan, C. (1996) A Simple Transformation for Nonlinear Waves. Physics Letters A, 224, 77-84. https://doi.org/10.1016/S0375-9601(96)00770-0

[12] Donagi, R. and Witten, E. (1996) Supersymmetric Yang-Mills Theory and Integrable Systems. Nuclear Physics B, 460, 299-304.

https://doi.org/10.1016/0550-3213(95)00609-5

[13] Witten, E. (1991) Two Dimensional Gravity and Intersection Theory on Moduli Space. Survey in Differential Geometry, 1, 243-249. https://doi.org/10.4310/SDG.1990.v1.n1.a5

[14] Chadan, K. and Sabatier, P.C. (1977) Inverse Problem in Quantum Scattering Theory. Springer, New York. https://doi.org/10.1007/978-3-662-12125-2

[15] Ablowitz, M.J., Kaup, D.J., Newell, A.C. and Segur, H. (1974) The Inverse Scattering Transform-Fourier Analysis for Nonlinear Problems. Studies in Applied Mathematics, 53, 249-256. https://doi.org/10.1002/sapm1974534249

[16] Yang, C.N. and Mills, R.L. (1954) Conservation of Isotopic Spin and Isotopic Gauge Invariance. Physical Review, 96, 191-206. https://doi.org/10.1103/PhysRev.96.191

[17] Atiyah, M.F. and Ward, R.S. (1977) Instants and Algebraic Geometry. Communications in Mathematical Physics, 55, 117-129. https://doi.org/10.1007/BF01626514

[18] Khater, A.H., Callebaut, D.K. and Sayed, S.M. (2006) Exact Solutions for Some Nonlinear Evolution Equations Which Describe Pseudo-Spherical Surfaces. Journal of Computational and Applied Mathematics, 189, 87-394. https://doi.org/10.1016/j.cam.2005.10.007

[19] Ablowitz, M.J., Chakravarty, S. and Halburd, R. (1998) The Generlized Chazy Equation and Schwarzian Triangle Functions. Asian Journal of Mathematics, 2, 619-627. https://doi.org/10.4310/AJM.1998.v2.n4.a1

[20] Ablowitz, M.J., Chakravarty, S. and Halburd, R. (1999) The Generalized Chazy Equation from the Self-Duality Equations. Studies in Applied Mathematics, 103, 75 85. https://doi.org/10.1111/1467-9590.00121

[21] Ablowitz, M.J., Chakravarty, S. and Halburd, R. (1998) On Painlevé and DarbouxHalphen Type Equations, in the Painlevé Property, One Century Later. Conte, R., Ed., CRM Series in Mathematical Physics, Springer, Berlin.

[22] Ablowitz, M.J., Chakravarty, S. and Halburd, R. (2003) Integrable Systems and Reductions of the Self-Dual Yang-Mills Equations. Journal of Mathematical Physics, 44, 3147-3153. https://doi.org/10.1063/1.1586967

[23] Khater, A.H., Callebaut, D.K. and Sayed, S.M. (2004) Conservation Laws for Some 
Nonlinear Evolution Equations Which Describe Pseudo-Spherical Surfaces. Journal of Geometry and Physics, 51, 332-352. https://doi.org/10.1016/j.geomphys.2003.11.009

[24] Ablowitz, M.J., Chakravarty, S. and Takhtajan, L.A. (1993) A Self-Dual Yang-Mills Hierarchy and Its Reductions to Integrable Systems in $1+1$ and $2+1$ Dimensions. Communications in Mathematical Physics, 158, 289-296. https://doi.org/10.1007/BF02108076

[25] Khater, A.H., Callebaut, D.K., Abdalla, A.A. and Sayed, S.M. (1999) Exact Solutions for Self-Dual Yang-Mills Equations. Chaos Solitons \& Fractals, 10, 1309-1320. https://doi.org/10.1016/S0960-0779(98)00155-6

[26] Khater, A.H., Callebaut, D.K., Abdalla, A.A., Shehata, A.M. and Sayed, S.M. (1999) Bäcklund Transformations and Exact Solutions for Self-Dual SU(3) Yang-Mills Equations, IL Nuovo Cimento B, 114, 1-10.

[27] Khater, A.H. and Sayed, S.M. (2002) Exact Solutions for Self-Dual SU(2) and SU(3) Yang-Mills Fields. International Journal of Theoretical Physics, 41, 409-419. https://doi.org/10.1023/A:1014241120146

[28] Khater, A.H., Shehata, A.M., Callebaut, D.K. and Sayed, S.M. (2004) Self-Dual Solutions for SU(2) and SU(3) Gauge Fields on Euclidean Space. International Journal of Theoretical Physics, 43, 151-159. https://doi.org/10.1023/B:IJTP.0000028857.57274.cd

[29] Sayed, S.M., Elkholy, A.M. and Gharib, G.M. (2008) Exact Solutions and Conservation Laws for Ibragimov-Shabat Equation Which Describe Pseudo-Spherical Surface. Computational \& Applied Mathematics, 27, 305-318. https://doi.org/10.1590/S0101-82052008000300005

[30] Sayed, S.M., Elhamahmy, O.O. and Gharib, G.M. (2008) Travelling Wave Solutions for the KdV-Burgers-Kuramoto and Nonlinear Schrödinger Equations Which Describe Pseudospherical Surfaces. Journal of Applied Mathematics, 2008, 576-583. https://doi.org/10.1155/2008/576783

[31] Khater, A.H., Callebaut, D.K. and Sayed, S.M. (2006) Exact Solutions for Some Nonlinear Evolution Equations Which Describe Pseudo-Spherical Surfaces. Journal of Computational and Applied Mathematics, 189, 387-411. https://doi.org/10.1016/j.cam.2005.10.007

[32] Sayed, S.M. and Gharib, G.M. (2009) Canonical Reduction of Self-Dual Yang-Mills Equations to Fitzhugh-Nagumo Equation and Exact Solutions. Chaos Solitons \& Fractals, 39, 492-498. https://doi.org/10.1016/j.chaos.2007.01.076

[33] Sayed, S.M. (2013) The Bäcklund Transformations, Exact Solutions, and Conservation Laws for the Compound Modified Korteweg-de Vries-Sine-Gordon Equations Which Describe Pseudospherical Surfaces. Journal of Applied Mathematics, 2013, 1-10. https://doi.org/10.1155/2013/613065

[34] Konno, K. and Wadati, M. (1975) Simple Derivation of Bäcklund Transformation from Riccati Form of Inverse Method. Progress of Theoretical Physics, 53, 1652 1655. https://doi.org/10.1143/PTP.53.1652

[35] Janusz, D. and Andrzej, S. (2017) Hamiltonian Paths in Hypercubes with Local Traps. Information Sciences, 375, 258-270. https://doi.org/10.1016/j.ins.2016.10.011

[36] Paul, C. and Olivier, F. (2017) On the Hamiltonian Structure of Large Deviations in Stochastic Hybrid Systems. Journal of Statistical Mechanics. Theory and Experiment, 2017, 534-542. 
Submit or recommend next manuscript to SCIRP and we will provide best service for you:

Accepting pre-submission inquiries through Email, Facebook, LinkedIn, Twitter, etc. A wide selection of journals (inclusive of 9 subjects, more than 200 journals)

Providing 24-hour high-quality service

User-friendly online submission system

Fair and swift peer-review system

Efficient typesetting and proofreading procedure

Display of the result of downloads and visits, as well as the number of cited articles Maximum dissemination of your research work

Submit your manuscript at: http://papersubmission.scirp.org/

Or contact ajcm@scirp.org 